\title{
A nano lamella NbTi-NiTi composite with high Strength
}

\author{
Jiang Jiang ${ }^{\mathrm{a}, \mathrm{b}^{*}}$, Daqiang Jiang ${ }^{\mathrm{b}}$, Shijie Hao ${ }^{\mathrm{b}}$, Cun Yu ${ }^{\mathrm{b}}$, Junsong Zhang ${ }^{\mathrm{b}}$, Yang Ren ${ }^{\mathrm{c}}$, Deping $\mathrm{Lu}^{\mathrm{a}}$, \\ Shifang $\mathrm{Xie}^{\mathrm{a}}$, and Lishan $\mathrm{Cui}^{\mathrm{b}}$ \\ ${ }^{a}$ Jiangxi Key Laboratory of Advanced Copper and Tungsten Materials and Institute of Applied Physics of Jiangxi \\ academy of sciences, Nanchang 330029 China \\ ${ }^{b}$ State Key Laboratory of Heavy Oil Processing and Department of Materials Science and engineering, China \\ University of Petroleum, Beijing 102249, China \\ ${ }^{c}$ X-ray Science Division, Argonne National Laboratory, Argonne, Illinois 60439, USA
}

Abstract: A hypereutectic $\mathrm{Nb}_{60} \mathrm{Ti}_{24} \mathrm{Ni}_{16}$ (at.\%) alloy was prepared by vacuum induction melting, and a nano lamellae NbTi-NiTi composite was obtained by hot-forging and wire-drawing of the ingot. Microscopic analysis showed that $\mathrm{NbTi}$ and NiTi nano lamellae distributed alternatively in the composite, and aligned along the wire axial direction, with a high volume fraction $(\sim 70 \%)$ of NbTi nano lamellae. In situ synchrotron X-ray diffraction analysis revealed that stress induced martensitic transformation occurred upon loading, which would effectively weaken the stress concentration at the interface and avoid the introduction of defects into the nano reinforced phase. Then the embedded NbTi nano lamellae exhibited a high elastic strain up to $2.72 \%, 1.5$ times as high as that of the $\mathrm{Nb}$ nanowires embedded in a conventional plastic matrix, and the corresponding stress carried by $\mathrm{NbTi}$ was evaluated as $2.53 \mathrm{GPa}$. The high volume fraction of NbTi nano lamellae improved the translation of high strength from the nano reinforced phase into bulk properties of the composite, with a platform stress of $\sim 1.7 \mathrm{GPa}$ and a fracture strength of $\sim 1.9 \mathrm{GPa}$.

\footnotetext{
* Corresponding author. Tel: +86-0791-88176237; superji1981@163.com (J. Jiang)
} 
Key words: Nano lamellae composite; Martensitic transformation; Shape memory alloy (SMA); NiTi; In situ synchrotron X-ray diffraction

\section{Introduction}

For a long time, the mechanical performances of nanostructure composites are far below what we have expected, primarily because the remarkable properties of the nano reinforced phase can not be exploited successfully in bulk composites [1-4]. In the case of metal matrix nanocomposite, the problem is associated with the stress concentration caused by the dislocation accumulation at the interface [1], which would lead to an earlier plastic deformation or fracture of the nano reinforced phase before its intrinsic strength was exhibited. Therefore, it seems impossible to translate the high strength of the nano reinforced phase into bulk strength of a metal matrix composite. However, this stereotype was broken by a recent work published in Science [1]. The study proposed that when NiTi shape memory alloy (SMA) was adopted as the matrix, the embedded nanowire could exhibit an ultra-large elastic strain comparable to that of a freestanding nanowire (4 to $7 \%$ ) [5-11], as the main deformation mechanism of the SMA was martensitic transformation rather than dislocation slipping upon loading. Then exceptional mechanical performances of the composite, such as high strength and large elastic strain were achieved [1]. As a result, tailoring and obtaining superior structural-functional properties by combining SMA with other reinforced phase in micro/nano scale, has become a new focus in materials science and design researches [1, 12-22].

Recently proposed $\mathrm{Nb}$ nanowire reinforced NiTi SMA composites were prepared 
by hot working and cold working of hypo eutectic or eutectic NiTi-Nb alloys [1, $12-14,17,21]$. The microstructure of such as-cast alloy is the typical lamellar eutectic structure of $\mathrm{NbTi}$ and $\mathrm{NiTi}$, and the average thickness of NbTi phase is less than 1 micrometer, which make it easier to form a $\mathrm{Nb}$ nanowire by drawing. However, the $\mathrm{Nb}$ fraction at the eutectic point is just $20 \%$ (at.\%) according to the $\mathrm{NiTi}-\mathrm{Nb}$ pseudobinary phase diagram [23], and therefore the max volume fraction of the embedded nanowire was not more than $\sim 25 \%$. One can assume that if the nanowire fraction could be significantly larger than that of SMA, the translation of high strength from the nano reinforced phase into bulk strength would be further improved, and therefore the performance of the bulk composite would be much closer to that of every embedded high performance nanowire. In this work, a nano lamellae NbTi-NiTi composite was fabricated by hot-forging and hot-drawing of a hypereutectic $\mathrm{NiTi}-\mathrm{Nb}$ alloy. Although the scales of most NbTi phase in the hypereutectic ingot are tens or even hundreds of times larger than that in a hypo eutectic or eutectic NiTi-Nb ingot, as a result of the increase of $\mathrm{Nb}$, nano scale $\mathrm{NbTi}$ lamellae were still obtained after hot working, and the volume fraction of $\mathrm{NbTi}$ nano lamellae was up to $70 \%$. Extraordinary mechanical properties of the composite as well as high elastic strain of the NbTi nano lamellae were obtained.

\section{Experimental}

$\mathrm{Nb}_{60} \mathrm{Ti}_{24} \mathrm{Ni}_{16}$ (at.\%) alloy ingot of $6 \mathrm{~kg}$ was prepared by vacuum induction melting. The obtained ingot was hot forged at $850^{\circ} \mathrm{C}$ into a rod with $8 \mathrm{~mm}$ in diameter, and then was hot drawn at $600^{\circ} \mathrm{C}$ into a wire with $0.5 \mathrm{~mm}$ in diameter. All 
test samples cut from the wire were annealed at $450^{\circ} \mathrm{C}$ for 20 min and followed by air cooling. A scanning electron microscope (SEM, FEI Quanta 200F) equipped with an energy dispersive X-ray (EDX) analyzer, and a transmission electron microscopy (TEM, FEI Tacnai F20) together with high angle angular dark field scanning transmission electron microscopy (HAADF STEM) were employed to examine the sample. Tensile tests were conducted on a WDT II-20 testing machine with a strain rate of $1 \times 10^{-3} \mathrm{~s}^{-1}$ at room temperature. In situ synchrotron $\mathrm{x}$-ray diffraction measurements were performed at the 11-ID-C beamline of the Advanced Photon Source, Argonne National Laboratory. High energy x-rays having a beam size of 0.6 $\mathrm{mm} \times 0.6 \mathrm{~mm}$ and wavelength of $0.010798 \mathrm{~nm}$ were used to obtain two-dimensional (2-D) diffraction patterns in the transmission geometry.

\section{Results and discussion}

\section{Figure 1.}

Figure 1a shows the microstructure of the as-cast $\mathrm{Nb}_{60} \mathrm{Ti}_{24} \mathrm{Ni}_{16}$ alloy ingot. The bright areas are bcc-NbTi primary phase, with about tens of micrometers size, and the darker areas are eutectic structure of $\mathrm{NbTi}$ and near-equiatomic TiNi. The volume fraction of NbTi phase and NiTi phase is about $70 \%$ and $30 \%$ respectively. After the ingot was hot-forged and drawn into a wire, this microstructure became much thinner and elongated along the drawing direction, which seems as a continuous fiber reinforced composite, as shown in Figure $1 \mathrm{~b}$ (cross section) and Figure 1c (longitudinal section). One can see from Figure 1c that the thickness of both NbTi phase (brighter contrast area) and NiTi phase (darker contrast area) are in nano scale. 
This indicates that each bright contrast area (Nb-rich phase) with micron or submicron size in Figure $1 \mathrm{~b}$ is not a single NbTi phase, but a mixture of alternatively distributed crimped NbTi and NiTi lamellae. The high volume fraction of Nb-rich phase shown in Figure $1 \mathrm{~b}$ means that almost the whole wire is composed of such nano lamellae structure. The 2-D high-energy x-ray diffraction pattern of the specimen (inset in Figure 1c) can be indexed to body-centered cubic NbTi (marked by "Nb") and B2-NiTi phase (marked by "B2"), in which strong texture of NbTi (110) planes perpendicular to the axial direction of the wire (vertical direction of the inset) was observed.

\section{Figure 2.}

Figure 2a shows the tensile curves of the composite wire, which exhibits a high mechanical performance. The fracture strength and fracture strain of the sample are $1940 \mathrm{MPa}$ and $14.9 \%$ respectively, and the starting stress of the yield platform is up to $1730 \mathrm{MPa}$. It is rare for a nanostructure composite to simultaneously exhibit a high yield stress over $1700 \mathrm{MPa}$ and a high plasticity ( 15\% fracture strain), as high fraction brittle nanoreinforcement often leads to a high strength but poor plasticity.

The inset in Figure 2a shows multiple-step tensile cyclic tests of the sample. One can see that the sample can recover completely when was loaded to a strain of $1 \%$, whereas a residue strain of $0.25 \%$ was observed in the tensile cycle with the max strain of $2 \%$. Therefore, elastic-plastic deformation started to occur within the strain range of $1 \%$ and $2 \%$. When tensile testing a pre-strained sample which was subjected to a $5 \%$ max strain pre-tensile cycle, most plastic deformation occurred before the stress platform was wiped out, as shown in Figure 2b. One can see from the inset that 
the sample can recover completely when was unloaded from the strain of $2 \%$. In the tensile cycle with the max strain of $2.7 \%$, the residue strain is just $0.15 \%$ after unloading. Then one can conclude that the elastic strain limit of the sample is about $2.7 \%$.

Figure 3.

In situ synchrotron x-ray diffraction was carried out on the sample to investigate the deformation behavior of all components inside the composite during the tensile test. Figure $3 \mathrm{a}$ shows the evolution of the diffraction patterns containing the peaks corresponding to $\mathrm{B} 2-\mathrm{NiTi}$ (110), $\mathrm{NbTi}$ (110) and $\mathrm{B} 19^{`}-\mathrm{NiTi}$ (001) planes perpendicular to the loading direction. Upon loading (from the spectrum at the bottom to top), the shift of the peaks to larger $d$-spacing means the elastic strain of $\mathrm{NbTi}$ and NiTi nano lamellae inside the composite gradually increased along the wire axial direction (loading direction). The decrease in intensity of the B2-NiTi (110) peaks and the concomitant increase in intensity of the B19`-NiTi (001) peaks indicate that stress induced martensitic transformation (B2-NiTi austenite phase transformed to B19`-NiTi martensite phase) took place during the loading process.

Detailed evolution of all detected B2-NiTi (110) planes during transformation process is shown in Figure 3b. The split of the peaks upon loading is associated with the elongation in axial direction and shrinkage in radial direction of the wire. The integrated peak area of these B2 (110) planes has been plotted as a function of the applied macro-strain, as shown in Figure $3 \mathrm{c}$, and the relative change in integrated areas of B2 peaks indicates the transformed volume fraction for NiTi $[1,24]$. One can see that the peak areas decrease almost linearly from $0 \%$ to $4 \%$ applied macro-strain, 
which means that the stress induced martensitic transformation starts even at the beginning of the tensile test, and the deformation of NiTi was dominated by transformation within a $\sim 4 \%$ macro-strain range. The B2 peaks became undistinguishable when the strain exceeded $5 \%$.

\section{Figure 4.}

In order to characterize the elastic deformation behavior of the $\mathrm{NbTi}$ nano lamellae inside the composite more visually, plots of lattice strain for these NbTi (110) planes versus macroscopic strain are given in Figure 4 (circular plots). The lattice strain was calculated using $\left(d_{h k l}-d_{h k l}^{0}\right) / d_{h k l}^{0}$, where $d_{h k l}$ is the $d$-spacing value corresponds to each of the $\operatorname{NbTi}(110)$ peaks in Figure $3 \mathrm{a}$, and $d_{h k l}^{0}$ is the $d$-spacing value of $\mathrm{NbTi}$ (110) peak before loading. As these NbTi (110) planes are perpendicular to the loading direction, one can regard the $d$-spacing strain given in Figure 4 as the axial elastic strain of $\mathrm{NbTi}$ nano lamellae $\left(\varepsilon_{\mathrm{NbTi}}\right)$, and the average stress carried by $\mathrm{NbTi}$ can be broadly evaluated using $\sigma_{\mathrm{NbTi}}=E_{<110, N b T i} \times \varepsilon_{\mathrm{NbTi}}$, where $E_{<11 \infty, N b T i}$ is the elastic modulus of the $<110>$ oriented NbTi nano lamellae (93 GPa [25] was adopted here). Then the stress-strain curve of NbTi nano lamellae inside the composite was obtained, as shown by the triangular plots, where the macro-strain of the wire was approximately considered as the total strain (elastic+plastic strain) of the NbTi nano lamellae. One can see that the max elastic strain ( $d$-spacing strain) of NbTi nano lamellae is $2.72 \%$, and the corresponding stress carried by $\mathrm{NbTi}$ nano lamellae is $2.53 \mathrm{GPa}$. Obviously, the high strength of the NbTi nano lamellae contributes to the high mechanical performance of the macro composite wire.

In most previous proposed conventional nanostructure composites, the max 
elastic strain of the embedded nanowire was less than $1.8 \%$, such as the typical high performance multiscale nanofilamentary $\mathrm{Cu}-\mathrm{Nb}$ composite [26-28]. Whereas in this work, the elastic strain of the NbTi nano lamellae was up to $2.72 \%, 50 \%$ higher than that of the $\mathrm{Nb}$ nanowires in conventional nanostructure composites. The advantage in this composite is the adoption of NiTi SMA as the second phase to combine with the NbTi nano reinforced phase. Due to the stress induced martensitic transformation occurred upon loading, NiTi phase generated a transformation strain rather than plastic deformation of dislocation slipping to coordinate with the macro-strain of the composite. Then the stress concentration caused by the dislocation accumulation at the interface, which would introduce defects (such as dislocation slipping) into nano reinforced phase and reduce its intrinsic properties, was avoided effectively. Therefore, the elastic strain of the nanoreinforcement was much improved, although the volume fraction of NiTi SMA was reduced to about $30 \%$.

\section{Conclusion}

To summarize, based on the design strategy that a transforming second phase material would weaken the stress concentration at the interface, and improve the reinforcing effect of nano components, a nano lamellae NbTi-NiTi composite with high volume fraction of NbTi nanoreinforcement (up to $~ 70 \%$ ) was prepared. The microstructure of the obtained $\mathrm{Nb}_{60} \mathrm{Ti}_{24} \mathrm{Ni}_{16}$ wire is quite similar to a continuous fiber composite, with alternatively distributed nano thick $\mathrm{NbTi}$ and $\mathrm{NiTi}$ lamellae aligning along the drawing direction. As stress induced martensitic transformation of NiTi occurred during the tensile test, a high elastic strain $(2.72 \%)$ of the $\mathrm{NbTi}$ nano 
lamellae was obtained, corresponding to a stress up to $2.53 \mathrm{GPa}$. The high volume fraction of $\mathrm{NbTi}$ nanoreinforcement further improved the translation of high strength from the nano reinforced phase into bulk strength, which contributes to the high performance of the macro composite wire.

\section{Acknowledgement}

This work is supported by the key program project of National Natural Science Foundation of China (NSFC) (51231008, and 51401096), the Key Project of Chinese Ministry of Education (313055), the Ph. D Programs Foundation of Jiangxi Academy of Sciences (No. 2013-YYB-5), and the Special Funds for Collaborative Innovation of Jiangxi Academy of Sciences (No. 2013-XTPH1-33). The use of the Advanced

Photon Source was supported by the US Department of Energy, Office of Science, and Office of Basic Energy Science under Contract No. DE-AC02-06CH11357.

\section{References}

[1] S.J. Hao, L.S. Cui, D.Q. Jiang, et al., Science, 339 (2013) 1191.

[2] Y. Dzenis, Science 319 (2008) 419.

[3] P. Podsiadlo AK Kaushik, EM Arruda, et al., Science 318 (2007) 80.

[4] J. N. Coleman, U. Khan, Y. K. Gun'ko, Adv. Mater. 18, (2006) 689.

[5] E. W. Wong, P. E. Sheehan, C. M. Lieber, Science 277 (1997) 1971.

[6] T. Zhu, J. Li, Prog. Mater. Sci. 55 (2010) 710.

[7] Y. Yue, P. Liu, Z. Zhang, X. Han, E. Ma, Nano Lett. 11 (2011) 3151. 
[8] G. Richter et al., Nano Lett. 9 (2009) 3048.

[9] L. Tian et al., Nat Commun. 3 (2012) 609.

[10] K. Koziol et al., Science 318 (2007) 1892.

[11] D. A. Walters et al., Appl. Phys. Lett. 74 (1999) 3803.

[12] S.J. Hao, L.S. Cui, Y.D. Wang, et al., Appl. Phys. Lett. 99 (2011) 024102.

[13] S.J. Hao, D.Q. Jiang, L.S. Cui, et al., Appl. Phys. Lett. 99 (2011) 084103.

[14] S.J. Hao, L.S. Cui, D.Q. Jiang, et al., Appl. Phys. Lett. 101 (2012) 13115.

[15] S.J. Hao, L.S. Cui, Y. Shao, et al., Appl. Phys. Lett. 101 (2012) 221904.

[16] S.J. Hao, L.S. Cui, Z.H. Chen, et al., Adv. Mater. 25 (2013) 1199.

[17] Z.Y. Liu, L.S. Cui, Y.N. Liu, et al., Scr. Mater. 77 (2014) 75.

[18] S. Wang, F.M. Guo, D.Q. Jiang, Y. Liu and L.S. Cui, Scr. Mater. 81 (2014) 4.

[19] J.S. Zhang, Y.N. Liu, Y. Ren, et al., Appl. Phys. Lett. 105 (2014) 041910.

[20] J.S. Zhang, Y.N. Liu, Y. Huan, et al., Mater. Des. 63 (2014) 460.

[21] Z.Y. Liu, Y.N. Liu, D.Q. Jiang, et al., Mater. Sci. Eng., A 610 (2014) 6.

[22] D.Q. Jiang, S.J. Hao, J.S. Zhang, et al., Scr. Mater. 53 (2014) 78.

[23] M. Piao, S. Miyazaki, K. Otsuka, N. Nishida. Mater.Trans. JIM. 33 (1992) 337.

[24] J. Mu, Z.W. Zhu, R. Su, et al., Acta Mater. 61 (2013) 5008.

[25] D. E. Cohen and J. Bevk, Appl. Phys. Lett. 39 (1981) 595.

[26] L. Thilly, P. O. Renault, and V. Vidal, Appl. Phys. Lett. 88 (2006) 191906.

[27] L. Thilly, S. Van Petegem, P.-O. Renault, et al., Acta Mater. 57 (2009) 3157.

[28] V. Vidal, L. Thilly, S. Van Petegem, et al., Scr. Mater. 60 (2009) 171. 


\section{Figure Captions}

Figure 1. (a) $\mathrm{SEM}$ micrograph of the as-cast $\mathrm{Nb}_{60} \mathrm{Ti}_{24} \mathrm{Ni}_{16}$ alloy ingot. The $\mathrm{NbTi}$ phases show brighter contrast and eutectic structures (NiTi+NbTi) seem relatively darker. (b) SEM micrograph of the cross-section of the annealed $\mathrm{Nb}_{60} \mathrm{Ti}_{24} \mathrm{Ni}_{16}$ wire. (c) STEM image of the longitudinal section of the wire, with NbTi and NiTi nano lamellae align along the axial direction of the wire. The inset in (c) is the 2-D high-energy x-ray diffraction pattern of the wire, and the vertical direction is the axial direction of the wire.

Figure 2. (Color online) (a) Tensile stress-strain curve of the sample. The inset in (a) shows multiple-step cyclic stress-strain curves of the sample, and the max strains of each cycle were $1 \%, 2 \%, 3 \%$ and $5 \%$ in sequence. (b) Multiple-step cyclic stress-strain curves of a pre-strained sample (the sample was pre-loaded to a strain of $5 \%$, and the residue strain was $2.3 \%$ after unloading before tensile tests). The max strains of each cycle were $1 \%, 2 \%, 2.7 \%$ and fracture strain in sequence. The inset in (b) is an enlarged view of the first three multiple-step cycles in (b).

Figure 3. (Color online) (a) Evolution of the diffraction peaks of B2-NiTi(110), Nb (110) and B19`-NiTi (001) planes perpendicular to the loading direction during the tensile test. As the B19`-NiTi (001) peaks are too low and not quite clear, these curves were evaluated by Gaussian fitting. (b) Detailed evolution of diffraction peaks corresponding to all detected B2 NiTi (110) planes during the transformation process 
within the macro strain range from $0 \%$ to $8 \%$. (c) The relation between the integrated areas of the B2 NiTi (110) peaks shown in (b) and the corresponding applied macro-strain of the wire.

Figure 4. (Color online) Circular plots show relation between the $d$-spacing strain of $\mathrm{Nb}$ (110) planes and the corresponding macroscopic strain of the wire, which can be regarded as elastic strain versus total strain (elastic+plastic strain) relation of the NbTi nano lamellae (the corresponding Y-axis is on the left); Triangular plots show the relation between stress carried by the NbTi nano lamellae and the corresponding macroscopic strain of the wire, which can be regarded as the stress-strain curve of the NbTi nano lamellae inside the composite (the corresponding Y-axis is on the right) 


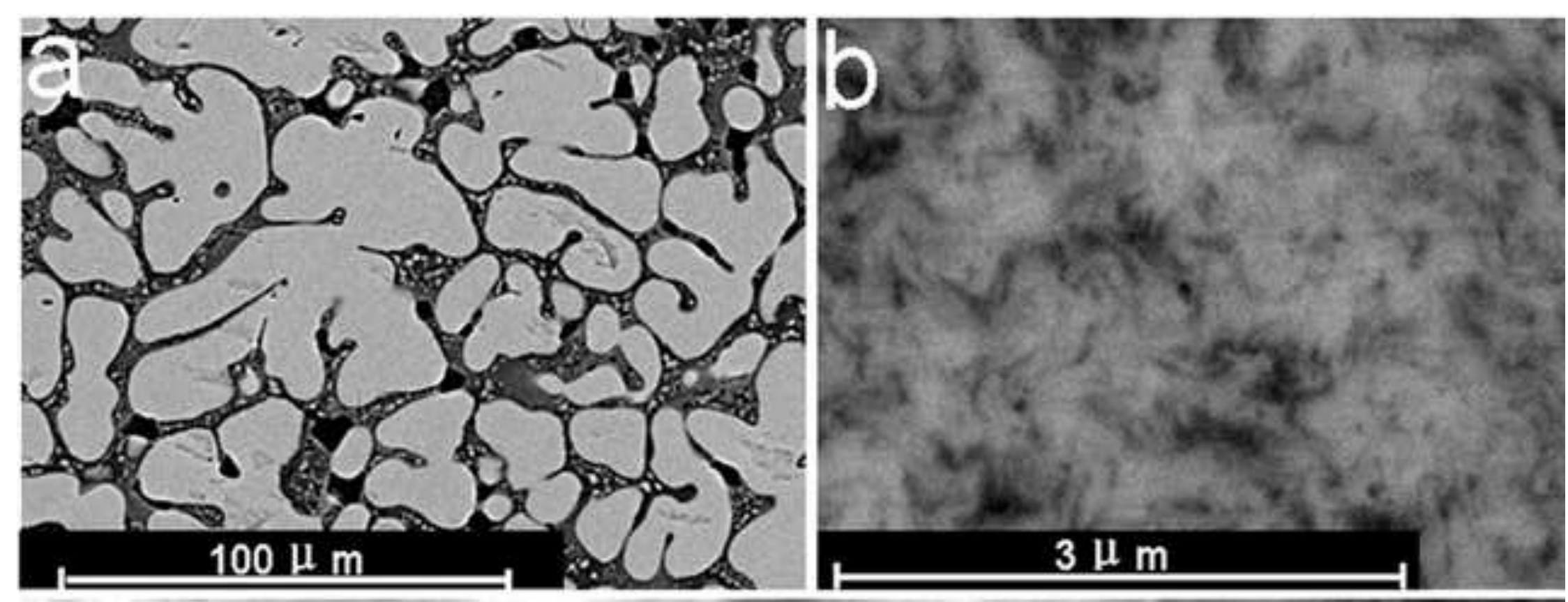

\section{C}

B2-NiTi nano lamella NbTi nano lamella

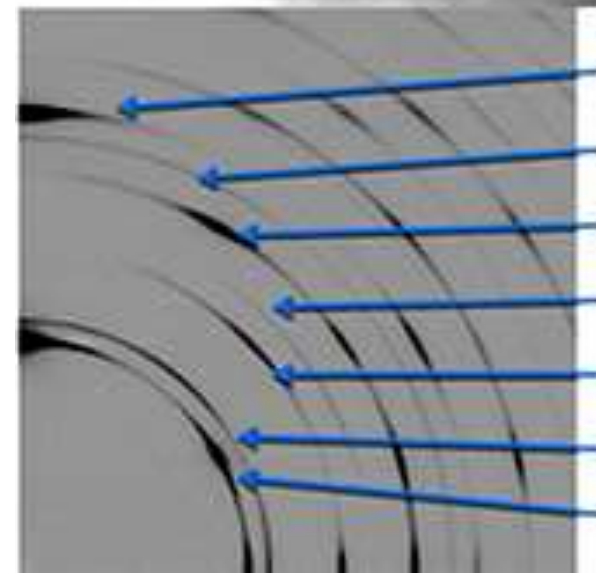

$\mathrm{Nb}$ (220)

B2 (211)

$\mathrm{Nb}$ (211)

B2 (200)

$\mathrm{Nb}(200)$

B2 (110)

$\mathrm{Nb}$ (110)

$200 \mathrm{~nm}$ 

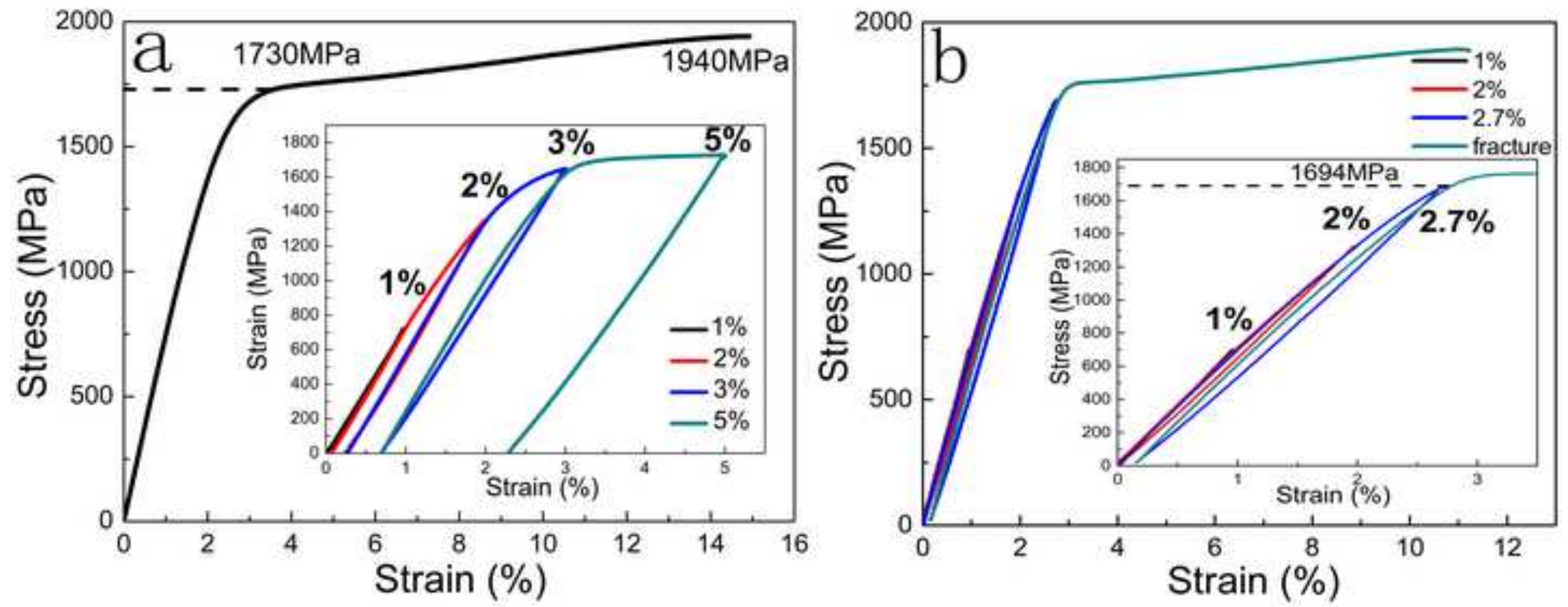

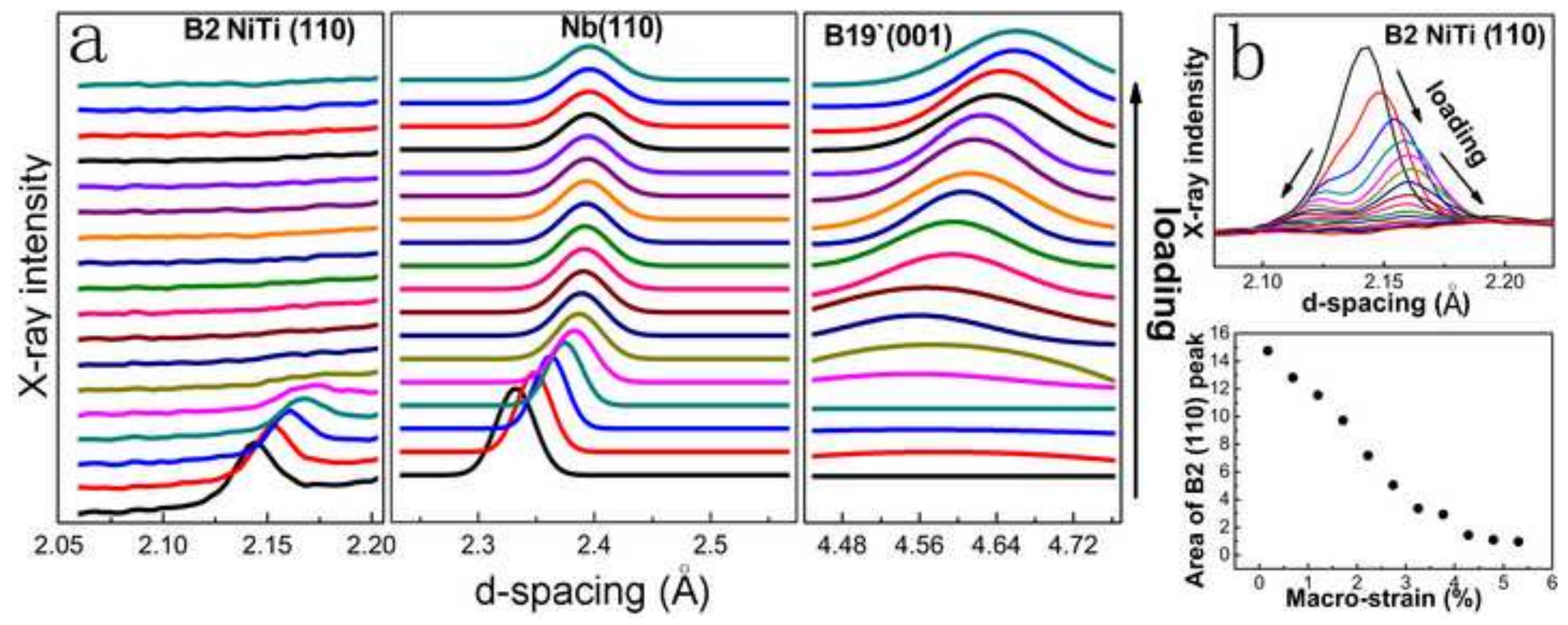


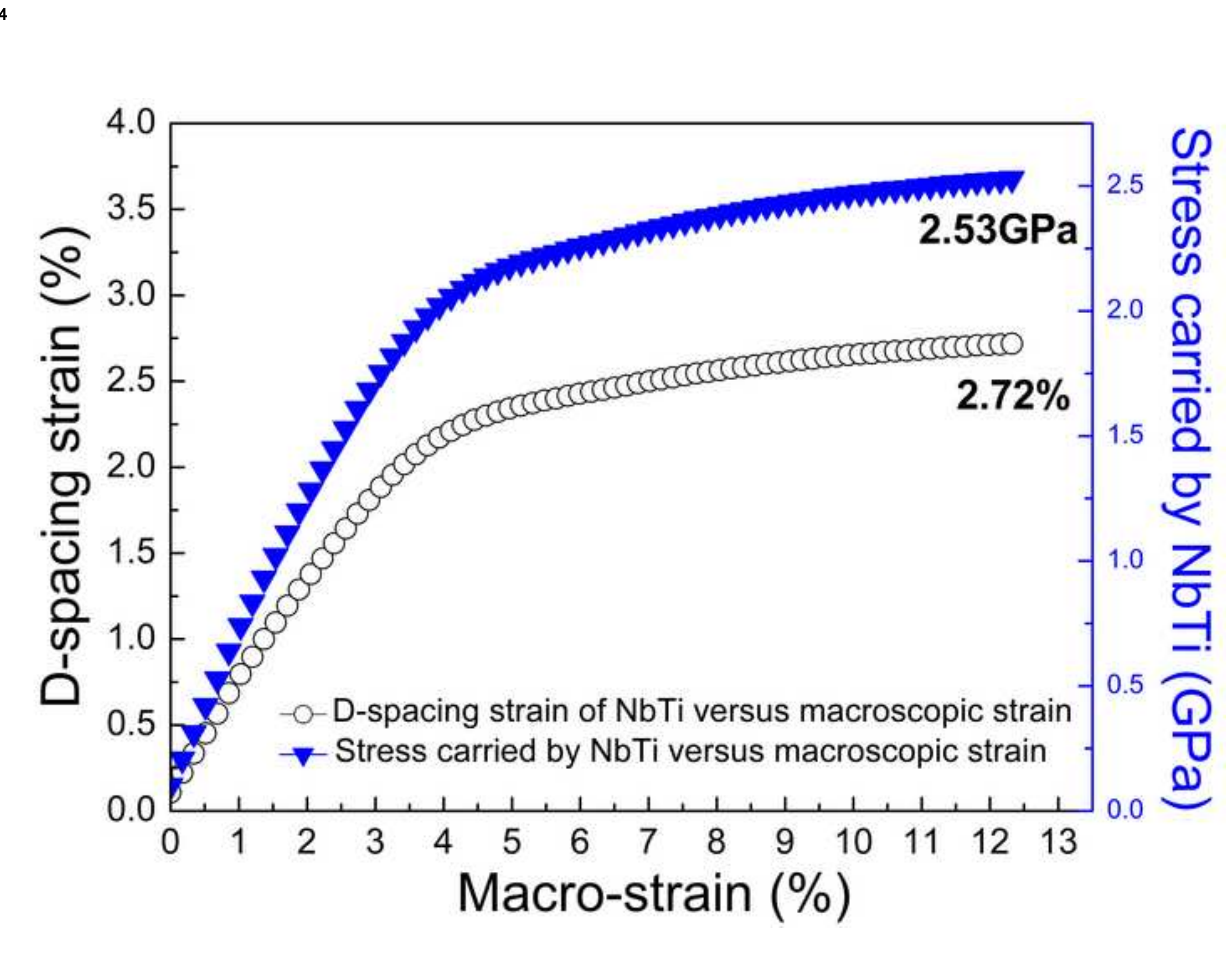

.

